\title{
Senotherapeutics for healthy ageing
}

Laura J. Niedernhofer and Paul D. Robbins

The recent manuscript by Childs et al. in Nature Reviews Drug Discovery ${ }^{1}$ thoroughly reviewed the important role senescent cells play in driving ageing and age-related diseases. The review also highlighted the clinical importance of developing senotherapeutic approaches to selectively kill senescent cells (senolytics) or to suppress the senescenceassociated secretory phenotype (SASP) that drives sterile inflammation associated with ageing (senomorphics), in order to extend healthspan and potentially lifespan. Clearly, senotherapeutic approaches can revolutionize how age-related diseases and ultimately how ageing itself can be treated. Given how quickly the field of senotherapeutics is moving towards clinical trials, we would like to expand upon several important issues regarding the current and future development of senotherapeutics. This is particularly important since the nascent field of senotherapeutics is faced with the challenge of establishing important standards and criteria for documenting a compound's or a combination of compounds' function as advertised, that is, as senotherapeutics.

It is established that senescent cells play a causative role in ageing and age-related disease. Therefore, the development of drugs that specifically kill senescent cells is envisioned to have significant therapeutic effects on slowing ageing phenotypes, treating age-related comorbidities and improving resiliency. However, not all senescent cells are the same, expressing different senescence markers, secreting different SASP factors and, more importantly, using different senescent cell anti-apoptotic pathways (SCAPs) to resist apoptosis. The elimination of senescent cells from multiple tissues or even a single tissue will probably require the combination of multiple senotherapeutic drugs ${ }^{2}$.

To date, seven classes of compound with some evidence of senolytic activity have been reported (see Supplementary Table 1), including the combination of dasatinib and quercetin, as well as BCL2 family inhibitors, identified using a bioinformatics approach for SCAPs ${ }^{2}$. In addition, a forkhead box protein $\mathrm{O} 4$ (FOXO4)-interacting peptide, which blocks the association of FOXO4 with p53, induces apoptosis of senescent human cells in vitro and reduces the expression of senescence markers while extending healthspan in vivo.
The natural compounds fisetin ${ }^{3}$, a quercetinrelated flavonoid, and piperlongumine also exhibit evidence of senolytic or senomorphic activity in certain cell types in vitro. Clinically used compounds targeting the co-chaperone heat shock protein 90 (HSP90) were also identified as a novel class of potential senolytics, able to induce apoptosis of senescent murine and human cells in vitro and improve healthspan in vivo ${ }^{4}$. Finally, the FDA-approved histone deacetylase inhibitor panobinostat induces apoptosis of senescent tumour cells in vitro. Clearly, there are multiple SCAP targets and thus it is highly likely that additional classes of potential senolytics will be identified through bioinformatic analyses and drugscreening approaches.

Several classes of senomorphics - drugs that suppress markers of senescence or their secretory phenotype without inducing apoptosis - have also been identified. Senomorphics include inhibitors of ІкB kinase (IKK) and nuclear factor (NF)- $\mathrm{KB}^{5}$, free radical scavengers and Janus kinase (JAK) pathway inhibitors $^{6}$. Even rapamycin acts as a senomorphic by reducing the SASP. Some compounds, for example fisetin, have senomorphic effects on some cell types while having senolytic activity on others, at least in vitro ${ }^{3}$.

It is important to note that definitively demonstrating that a drug is senolytic or senomorphic is challenging and should be approached critically. Simply measuring a reduction in senescence markers (such as the expression of $\mathrm{p} 16^{\mathrm{INK} 4 \mathrm{~A}}$ or $\mathrm{p} 21^{\mathrm{CIP} 1}$ or SASP factors) or in senescence-associated $\beta$-galactosidase (SA- $\beta$-gal) activity is inadequate to distinguish between the two mechanisms of action. Careful determination of whether there is selective loss of senescent cells is required. Notably, the assay selected to test potential therapeutics influences the outcome. For example, the cell type selected and the method used to induce senescence influences which SCAPs are expressed and therefore whether a particular class of drugs (for example, BCL2 inhibitors) will be effective in killing cells. Also, the end points measured will influence interpretation (for example, senescence markers, SASP, number of viable cells and/or apoptosis markers) as well as what control cells are used (for example, proliferating or quiescent). Thus, the assays used and end points measured should be taken into careful consideration when defining a senolytic or senomorphic drug and the likelihood of its broad efficacy.

Once evidence is found for senolytic activity of a compound in vitro, it is even more difficult to document potential senolytic activity in vivo (TABLE 1). Demonstrating that a compound reduces the number of SA- $B-$ gal $^{+}$ cells or the level of $p 16^{\text {ink } 4 a}$ expression, such as through the use of $p 16^{i n k 4 a}$-luciferase reporter mice, does not definitively demonstrate killing, let alone selective killing, of senescent cells. Also, since senolytics are cell type-specific, the lack of an effect on a specific tissue doesn't preclude senolytic activity in other tissues. Even more rigorous approaches, such as the injection of labelled senescent cells followed by drug treatment, only serve to document that the putative senolytic is functioning in vivo similar to in vitro, that is, by killing that specific type of transplanted senescent cell. Eventually, improved methods for colocalization of apoptosis and senescence in vivo at the single cell level, such as through the use of cytometry by time-of-flight (CyTOF) or dual fluorescent reporters, will be needed to define a compound as senolytic.

One current tactic to provide evidence that a compound has senolytic activity is the 'hit-and-run' approach, for example, the onetime treatment of mice with dasatinib and quercetin following hind-leg irradiation ${ }^{3}$. Here, a single administration of dasatinib plus quercetin yielded a therapeutic benefit that endured for months in terms of treadmill performance, consistent with a senolytic mechanism in which disease-causing cells are killed. In general, if a short course of treatment yields a sustained reduction in senescence, it is likely acting in a senolytic manner. In contrast, if chronic treatment is needed to suppress senescence markers or prevent secondary senescence, this is more consistent with senomorphic activity. However, more refined approaches are needed to rapidly demonstrate senolytic activity in vivo. Likely combinations of approaches may be necessary. For example, if a drug kills senescent endothelial cells, it could yield a health dividend, but it will be very difficult to document a reduction in the expression of senescence markers specifically in the endothelium without sophisticated reporter systems.

Another current approach to provide evidence that a compound potentially has senolytic activity in vivo is to compare the therapeutic efficacy of the drug to outcomes yielded by genetic ablation of senescent cells in transgenic INK-ATTAC or p16-3MR mice $^{7,8}$. For example, in the bleomycin mouse model of idiopathic pulmonary fibrosis, the 
Table 1 | Approaches for demonstrating the mechanism of action of senotherapeutics.

\begin{tabular}{|c|c|c|c|}
\hline \multirow[t]{2}{*}{ System for testing } & \multicolumn{2}{|l|}{ Class of drug } & \multirow[t]{2}{*}{ New methodologies } \\
\hline & Senolytic & Senomorphic & \\
\hline $\begin{array}{l}\text { p16-reporter mice and/or } \\
\text { measuring senescence in } \\
\text { multiple tissues using multiple } \\
\text { end points (expression of p16, } \\
\text { p21 and SASP, and/or decreased } \\
\text { SA-ß-gal activity) }\end{array}$ & $\begin{array}{l}\text { Short-term administration of a } \\
\text { senolytic must yield a sustained } \\
\text { reduction in senescence signal }\end{array}$ & $\begin{array}{l}\text { Chronic treatment with a } \\
\text { senomorphic is required for } \\
\text { a sustained suppression in } \\
\text { senescence signal }\end{array}$ & $\begin{array}{l}\text { - Additional transgenic reporters for } \\
\text { markers of senescence such as p21 } \\
\text { - Dual reporters for apoptosis and } \\
\text { senescence } \\
\text { - Analysis of activity in } \\
\text { immune-deficient mice }\end{array}$ \\
\hline $\begin{array}{l}\text { Human tissue explants } \\
\text { harbouring senescent cells }\end{array}$ & $\begin{array}{l}\text { Drug treatment yields a } \\
\text { reduction in senescence markers } \\
\text { (expression of p16, p21 and } \\
\text { SASP, and/or decreased SA-B-gal } \\
\text { activity) and an increase in } \\
\text { apoptosis markers }\end{array}$ & $\begin{array}{l}\text { Drug treatment yields a suppression } \\
\text { in senescence markers (expression } \\
\text { of p16, p21 and SASP, and/or } \\
\text { decreased SA-ß-gal activity) with no } \\
\text { evidence of cell death }\end{array}$ & $\begin{array}{l}\text { CyTOF, in situ hybridization and } \\
\text { related approaches to colocalize } \\
\text { downregulation of senescence } \\
\text { with upregulation of apoptosis } \\
\text { markers as well as cell identity } \\
\text { markers }\end{array}$ \\
\hline $\begin{array}{l}\text { Measure therapeutic efficacy in } \\
\text { aged or diseased organisms }\end{array}$ & $\begin{array}{l}\text { Short-term or intermittent } \\
\text { intervention with a senolytic must } \\
\text { yield a sustained health benefit in } \\
\text { old or diseased organisms }\end{array}$ & $\begin{array}{l}\text { Chronic administration of a } \\
\text { senomorphic is required to yield a } \\
\text { sustained health benefit in old or } \\
\text { diseased organisms }\end{array}$ & $\begin{array}{l}\text { Coordinate analysis of therapeutic } \\
\text { efficacy and a reduction of } \\
\text { senescence using bioluminescent } \\
\text { reporters or serum markers with } \\
\text { short or intermittent treatment in } \\
\text { models of natural and accelerated } \\
\text { ageing }\end{array}$ \\
\hline
\end{tabular}

Multiple systems are currently being used to test if a drug specifically targets senescent cells in vivo. Often what is reported is a reduction in senescence markers. To document that an intervention is truly senolytic will require additional lines of evidence including the development of new methods. These are outlined for several experimental systems. CyTOF, cytometry by time-of-flight; SA- $\beta$-gal, senescence-associated $\beta$-galactosidase; SASP, senescence-associated secretory

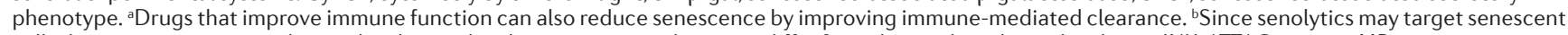
cells that are p16-negative, the results obtained with a putative senolytic may differ from the results achieved with p16-INK-ATTAC or p16-3MR mice.

therapeutic benefit of treatment with dasatinib and quercetin is comparable to clearing senescent cells in INK-ATTAC mice9. However, it will still be important to determine if the same cell types are targeted in the INK-ATTAC and the dasatinib and quercetin-treated mice. In fact, the mechanism of cell clearance in INKATTAC mice differs fundamentally from mice treated with senolytic agents. In the transgenic models, cells with high $p 16^{\text {ink4a }}$ promoter activity are targeted, while senolytics act by killing cells expressing specific pro-survival SCAPs. Importantly, not every cell with high $p 16^{\text {ink } 4 a}$ expression is senescent and not every senescent cell has high $p 16^{\text {ink4a }}$ expression. For example, $\mathrm{p} 16^{\mathrm{INK} 4 \mathrm{~A}}$ and SA- $\beta$-gal activity are induced in macrophages as part of a reversible response to physiological stimuli even though these cells are not senescent ${ }^{10}$.

An additional approach to provide evidence of senolytic activity in vivo involves the use of human tissue explants harbouring senescent cells ${ }^{7}$. A putative senolytic should attenuate senescence markers while inducing apoptosis. However, while this approach documents senolytic activity of a compound, it does not identify the types of cell undergoing cell death and whether it is only a subset of senescent cells. Additional methods, including flow cytometry and CyTOF-based approaches, are needed to define the cell types within a tissue explant that are targeted by senotherapeutics, as well as to colocalize markers of senescence and apoptosis.

Proving that a compound exhibits in vivo senolytic activity is further complicated by potential indirect effects on the immune system. Functional immune cells, including natural killer and $\mathrm{T}$ lymphocytes, can remove senescent cells. However, as the immune system ages, the ability to clear senescent cells wanes, contributing to the accumulation of senescent cells. It is possible that some drugs, for example, rapamycin, reduce the burden of senescent cells indirectly by improving immune cell function. Similarly, inhibiting the SASP might improve immune clearance of senescent cells. Thus, demonstrating that a drug directly targets senescent cells must address the possibility that the drug improves host clearance of senescent cells.

All in all, there is reason to be tremendously excited about the health and economic impact of senotherapeutic drugs. However, as noted, caution is warranted in interpreting the mechanism of action and relative specificity, selectivity and efficacy. Furthermore, there is still much to learn about how best to identify, characterize and apply these drugs to improve human health.

Laura J. Niedernhofer and Paul D. Robbins are at the Department of Molecular Medicine and The Center on Aging, The Scripps Research Institute, Jupiter, FL, USA.

e-mail:Iniedern@scripps.edu; probbins@scripps.edu

doi: $10.1038 /$ nrd. 2018.44 Published online 13 Apr 2018

\section{Acknowledgements}

This work was supported by National Institutes of Health grants P01-AG043376, U19-AG056278 and AG044376 and by the Glenn Foundation. 


\section{CORRESPONDENCE}

\section{Competing interests}

The authors declare no competing interests.

\section{Supplementary information}

Supplementary information is available for this paper at https://doi.org/nrd.2018.44

1. Childs, B. G. et al. Senescent cells: an emerging target for diseases of ageing. Nat. Rev. Drug Discov. 16, 718-735 (2017)

2. Zhu, Y. et al. The Achilles' heel of senescent cells: from transcriptome to senolytic drugs. Aging Cell 14 644-658 (2015).
3. Zhu, Y. et al. New agents that target senescent cells: the flavone, fisetin, and the BCL-XL inhibitors, A1331852 and A1155463. Aging 9, 955-963 (2017).

4. Fuhrmann-Stroissnigg, $\mathrm{H}$. et al. Identification of HSP90 inhibitors as a novel class of senolytics. Nat. Commun. 8, 422 (2017)

5. Tilstra, J. S. et al. NF-kappaB inhibition delays DNA damage-induced senescence and aging in mice. J. Clin. Invest. 122, 2601-2612 (2012).

6. Xu, M. et al. JAK inhibition alleviates the cellular senescence-associated secretory phenotype and frailty in old age. Proc. Natl Acad. Sci. USA 112 , E6301-E6310 (2015).
7. Jeon, O. H. et al. Local clearance of senescent cells attenuates the development of post-traumatic osteoarthritis and creates a pro-regenerative environment. Nat. Med. 23, 775-781 (2017).

8. Baker, D. J. et al. Clearance of p16Ink4a-positive senescent cells delays ageing-associated disorders. Nature 479, 232-236 (2011).

9. Schafer, M. J. et al. Cellular senescence mediates fibrotic pulmonary disease. Nat. Commun. 8, 14532 (2017).

10. Hall, B. M. et al. p16(Ink4a) and senescenceassociated beta-galactosidase can be induced in macrophages as part of a reversible response to physiological stimuli. Aging 9, 1867-1884 (2017). 